\title{
LÖSUNG EINES PROBLEMS BEZÜGLICH EINER CHARAKTERISIERUNG DES JACOBSONSCHEN RADIKALS
}

\author{
Von \\ F. SZÁSZ (Budapest) \\ (Vorgelegt von L. RÉDEI)
}

\section{§ 1. Einleitung}

Der Begriff des Radikals, als eines Ideals, welches das Maß der Singularität eines Ringes in irgendeinem Sinne kennzeichnet, spielt in der Ringtheorie bekanntlich eine wichtige Rolle. Das Radikal wurde zuerst für Algebren endlichen Ranges über dem Grundkörper definiert. Später wurden verschiedene Definitionen für das Radikal eines beliebigen (assoziativen) Ringes ohne Endlichkeitsbedingungen empfohlen, und es wurde auch eine allgemeine Radikaltheorie aufgebaut. Unter den verschiedenen Typen der Radikale zeigte sich das Jacobsonsche Radikal am meisten nutzbar (JACOBSON [6]).

Im Buch [4] (Seite 486, Theorem 22. 15. 3) von E. Hille ist bewiesen ${ }^{1}$ (1948), da $\beta$ der Frattinische Untermodul ${ }^{2} \Phi_{r}$ eines Ringes $A$, als eines $A$-Rechtsmoduls $A$, ein zweiseitiges Ideal von $A$ ist, für das $A J \subseteq \Phi_{r}$ gilt, wobei $J$ das Jacobsonsche Radikal von $A$ bezeichnet. Es gilt freilich auch $J A \subseteq \Phi_{i}$. Da offenbar $\Phi_{r} \subseteq J$ und $\Phi_{l} \leqq J$ bestehen, ergibt sich im speziellen Fall, wenn $A$ ein Linkseinselement hat (vgl. Fuchs [1]), bzw. $x \in A x$ für jedes $x \in A$ oder $A J=J$ gilt, $\Phi_{r}=J$. Neulich hat A. KerTész [7] dieses Resultat von Hille's Buch folgendermaßen verschärft: Das Jacobsonsche Radikal $J$ von $A$ ist die Menge derjenigen Elemente $x$ von $A$, für die $y x$ für jedes $y \in A$ aus jedem Erzeugendensystem von $A$, als von einem $A$-Rechtsmodul $A$, weggelassen werden kann. Mit diesem Resultat von Kertész [7] ist die folgende Behauptung äquivalent: Das Jacobsonsche Radikal $J$ eines Ringes $A$ ist maximal in der Menge derjenigen Ideale $K$ von $A$, für die $A K \subseteq \Phi_{r}$ gilt.

Ein Rechtsideal $R$ eines Ringes $A$ nennen wir quasimodular in $A$, wenn es zu jedem Element $x \in A$ mit $x \notin R$ ein Element $y(\in A, \notin R)$ mit $y x \notin R$ gibt. Offenbar ist jedes modulare Rechtsideal auch quasimodular in $A$. Aus der erwähnten Kertészschen Charakterisierung $J=\left\{x ; x \in A, A x \leqq \Phi_{r}\right\}$ folgt unmittelbar auch eine andere Kennzeichnung von KeRTÉsz [8] des Jacobsonschen Radikals $J$, nach der $J$ mit dem. Durchschnitt $D$ aller quasimodularen maximalen Rechtsideale $R$ des Ringes

${ }^{1}$ Mit anderen Bezeichnungen und Benennungen.

2 Die Frattinische Untergruppe $\Phi$ einer Operatorengruppe $G$ mit dem Operatorenbereich $\Omega$ ist die Menge derjenigen Elemente $g \in G$, für die $g \alpha$ für jedes $\alpha \in \Omega$ aus jedem Erzeugenden system von $G$ weggelassen werden kann. Damit ist äquivalent (vgl. z. B. M. HALl [3]), daß $\Phi$ der Durchschnitt aller maximalen zuläßigen Untergruppen von $G$ bzw. $\Phi=G$ ist, je nachdem, ob $G$ eine maximale zulässige Untergruppe besitzt, oder nicht. Ähnlich werden die (rechtsseitigen bzw. linksseitigen) Frattinischen Untermoduln $\Phi_{r}$ und $\Phi_{l}$ eines als $A$-Rechtsmodul bzw. $A$-Linksmodul betrachteten Ringes $A$, definiert. 
$A$ übereinstimmt. ${ }^{3}$ Die quasimodularen maximalen Rechtsideale spielen hiernach von dem Gesichtspunkt einer Charakterisierung des Jacobsonschen Radikals aus eine wichtige Rolle.

Im Buch [8] von A. Kertész ist folgendes Problem (in anderer Abfassung) aufgeworfen:

Gibt es einen Ring $A$, der ein quasimodulares maximales Rechtsideal $R$ besitzt, das in A nicht modular ist?

Einen (assoziativen) Ring $A$ nennen wir $\Omega$-Ring, wenn $A$ ein solches quasimodulare maximale Rechstideal $R$ besitzt, das in $A$ nicht modular ist. Die quasimodularen maximalen, aber nicht modularen Rechtsideale eines $\Omega$-Ringes werden ausgezeichnet genannt. Ein $\Omega$-Ring $A$ wird bezüglich eines ausgezeichneten Rechtsideales $R$ reduziert genannt, wenn $A$ kein von Null verschiedenes, in $R$ liegendes zweiseitiges Ideal besitzt.

Das Hauptziel dieser Arbeit ist der Beweis der Existenz von $\Omega$-Ringen, woraus die Lösung des erwähnten Problems von A. Kertész folgt (vgl. § 2). Aus diesem Existenzbeweis für $\Omega$-Ringe ergibt sich auch die Tatsache, daß die in [8] gegebene Charakterisierung für $J$, und zwar der Beweis von $D=J$, eine nicht nur formal, sondern auch inhaltlich neue Kennzeichnung für $J$ bedeutet. Weiterhin werden wir auch die wichtigsten Eigenschaften der $\Omega$-Ringe und der reduzierten $\Omega$-Ringe bestätigen (vgl. \& 3 und 4) und auch einige offene Fragen bezüglich der $\Omega$-Ringe erwähnen. Zum Abschluß folgen einige weitere Bemerkungen bezüglich des Jacobsonschen Radikals (vgl. § 5).

Hinsichtlich der nötigen Grundbegriffe verweisen wir (außer den Fußnoten) auf die Handbücher [2], [3], [6] und [9].

\section{$\S 2$. Die Existenz der $\Omega$-Ringe}

In diesem $\S$ beweisen wir durch eine explizite Konstruktion die Existenz von $\Omega$-Ringen, d. h. wir geben' eine Lösung des im $\S 1$ erwähnten Problems von A. KeRTÉSZ an. Insbesondere folgt aus der Existenz der $\Omega$-Ringe, daß die in [8] gegebene Charakterisierung für das Jacobsonsche Radikal auch inhaltlich (nicht nur gestaltich) neu ist.

Es gilt der folgende

Satz 2. 1. Für jede unendliche Mächtigkeit it gibt es einen $\Omega$-Ring $A$ mit einem ausgezeichneten Rechtsideal $R$, derart, da $\beta$ A eine Algebra mit $\operatorname{Rang} A=\operatorname{Rang} R=11$ über einem Primkörper ist.

${ }^{3}$ Im (bald erscheinenden) Buch [8] von A. KeRTész wird $D=J$ in einem solchen Satz bestätigt, der das Jacobsonsche Radikal durch eine Folge miteinander äquivalenter Bedingungen kennzeichnet, und dessen Beweis zyklisch ist. Es kann aber $D=J$ auch direkt bewiesen werden. Da $D \leqq J$ gilt, genügt es zeigen, daß $D \neq J$ unmöglich ist. Gibt es nämlich ein Element $j \in J$ mit $j \notin D$, so existieren ein quasimodulares maximales Rechtsideal $R$ und ein Element $a \in A$ mit $a j \notin R, a \notin R$. Wegen der Maximalität von $R$ in $A$ gibt es ein Element $b \in A$ mit $a+R=a j b+R$, woraus durch ein leichtes Rechnen $a \in R$, also ein Widerspruch zu $a \notin R$ folgt, denn $j b$ ist wegen $j \in J$ quasiregular. Gilt nämlich $j b+c-j b c=0$ mit einem $c \in A$, so ergibt sich $a=a-a(j b+c-j b c)=a(1-j b)-a(1-j b) c \in R$. Aus diesem Widerspruch erhält man $D=J$. 
BEweIs. Es seien $p=o$ oder $p$ gleich einer Primzahl, $K_{p}$ ein Primkörper, it eine unendliche Mächtigkeit, $\Gamma$ eine Index-Menge der Mächtigkeit $m, \delta_{\alpha \beta}$ das Kroneckersche Delta-Symbol, ${ }^{4} A$ eine Algebra über $K_{p}$ mit den Basiselementen $a_{\alpha}, r_{\beta \gamma}, s_{\varepsilon \eta \vartheta}(\alpha, \beta, \gamma, \varepsilon, \eta, \vartheta \in \Gamma)$ und $R$ die über $K_{p}$ mit sämtlichen $r_{\beta \gamma}, s_{\varepsilon \eta \vartheta}$ erzeugte Teilalgebra von $A$. Definieren wir die Multiplikation der Elemente der Basis durch die folgende Tabelle:

\begin{tabular}{|c|c|c|c|}
\cline { 2 - 4 } & $a_{\varepsilon}$ & $r_{\varepsilon \eta}$ & $s_{\varepsilon \eta \vartheta}$ \\
\hline$a_{\alpha}$ & $a_{\varepsilon}$ & $\delta_{\alpha \varepsilon} \cdot a_{\eta}$ & $\delta_{\alpha \varepsilon} \cdot a_{2}$ \\
\hline$r_{\alpha \beta}$ & $s_{\alpha \beta \varepsilon}$ & $\delta_{\beta \varepsilon} \cdot r_{\alpha \eta}$ & $\delta_{\beta \varepsilon} \cdot s_{\alpha \eta \vartheta}$ \\
\hline$S_{\alpha \beta \gamma}$ & $S_{\alpha \beta \varepsilon}$ & $\delta_{\gamma \varepsilon} \cdot s_{\alpha \beta \eta}$ & $\delta_{\gamma \varepsilon} \cdot s_{\alpha \beta \beta}$ \\
\hline
\end{tabular}

Diese Algebra ist offenbar monomial (im Sinne von RÉDEI [9]). Jedes Element von $A$ läßt sich in der Gestalt

$$
a=\sum_{i}^{*} \pi_{i} a_{\alpha_{i}}+\sum_{i, j}^{*} \varrho_{i j} r_{\alpha_{i} \beta_{j}}+\sum_{i, j, k}^{*} \sigma_{i j k} s_{\alpha_{i} \beta_{j} \gamma_{k}}
$$

darstellen, wobei $\pi_{i}, \varrho_{i j}, \sigma_{i j k} \in K_{p}$ und alle drei Summen $\sum^{*}$ endlich sind. Es gilt ferner $a \in R$ dann und nur dann, wenn in (*) $\pi_{i}=o$ für jedes $i$ besteht.

Auf Grund der Multiplikationstabelle kann bewiesen werden, daß die Multiplikation in $A$ assoziativ ist.

Es ist leicht einzusehen, daß die Teilalgebra $R$ ein Rechtsideal, und zwar ein maximales Rechtsideal des (ohne den Operatorbereich $K_{p}$ angesehenen) Ringes $A$ ist.

Aus der Multiplikationstabelle folgt nämlich unmittelbar, daß $R$ ein Rechtsideal in $A$ ist. Gilt nun $a \notin R$ für ein $a \in A$, so existiert in (*) ein $\pi_{i} \in K_{p}$ mit $\pi_{i} \neq 0$, woraus sich $a\left(\pi_{i}^{-1} \varrho r_{\alpha_{i} \beta}\right)=\varrho a_{\beta}+r^{\prime}\left(r^{\prime} \in R\right)$ für jedes $\beta \in \Gamma$ und $\varrho \in K_{p}$, folglich $a R+R=A$ ergibt. Also $R$ ist wirklich ein maximales Rechtsideal in $A$.

Es wird jetzt gezeigt, daß $R$ in $A$ nicht modular ist.

Es sei nämlich $a$ ein beliebiges Element von $A$. Ist $a \in R$, so ergibt sich wegen $(1-a) a_{\alpha}=a_{\alpha}-a a_{\alpha} \notin R$ gewiß $(1-a) A \Phi R$. Ist aber $a \notin R$, so gibt es in (*) ein $i$ mit $\pi_{i} \neq o$, woraus man $(1-a)\left(-\pi_{i}^{-1} r_{\alpha_{i} \beta}\right)=a_{\beta}+r^{\prime \prime} \notin R\left(r^{\prime \prime} \in R\right)$, folglich $(1-a) A \Phi R$ erhält. $R$ ist also gewiss nicht modular in $A$.

Wir beweisen, daß $R$ in $A$ ein quasimodulares Rechtsideal ist.

Es sei nämlich $a$ ein Element von $A$ mit $a \notin R$. Nehmen wir an, daß $a_{\alpha} a=r^{*} \in R$ für einen Index $\alpha \in \Gamma$ gilt. Dann hat $a$ offenbar eine Gestalt $a=\sum_{i} \pi_{i} a+r$ mit endlicher Summe $\sum$ und $r \in R$. Da $a_{\alpha} r=-\sum_{i} \pi_{i} a_{\alpha_{i}}+r^{*}$ ist, hat $r$ die Form

$$
r=\sum_{i}^{*} \sigma_{i} r_{\alpha \alpha_{i}}+\sum_{i, j}^{*} \tau_{i j} s_{\alpha \beta_{j} \alpha_{i}}+r_{\alpha}
$$

${ }^{4}$ Es gilt also $\delta_{\alpha \beta}=1$ für $\alpha=\beta$ und $\delta_{\alpha \beta}=o$ für $\alpha \neq \beta$. 
mit $\sigma_{i}, \tau_{i j} \in K_{p}$ und mit endlichen Summen $\Sigma^{*}$, weiterhin mit $r_{\alpha} \in R$ und $a_{\alpha} r_{\alpha} \in R$. Folglich gilt für $r_{\alpha}$ eine Darstellung:

$(* *) \quad r_{\alpha}=\sum_{i, j}^{*} \pi_{i j}\left(r_{\alpha \gamma_{i}}-S_{\alpha \beta_{j} \gamma_{i}}\right)+\sum_{i, j}^{*} \sigma_{i j}^{\prime} r_{\beta_{i} \gamma_{j}}+\sum_{i, j, k}^{*} \tau_{i j k}^{\prime} S_{\varepsilon_{i} \eta_{j} \theta_{k}}$,

wobei $\pi_{i j}, \sigma_{i j}^{\prime}, \tau_{i j k}^{\prime} \in K_{p}, \beta_{i} \neq \alpha, \varepsilon_{i} \neq \alpha$, und alle drei Summen $\sum^{*}$ endlich sind. Wegen der Endlichkeit dieser Summen und wegen $|\Gamma|=m$ gibt es einen Index $\varepsilon \in \Gamma$ mit $\varepsilon \neq \alpha$ und derart, daß $\varepsilon$ von sämtlichen in (***) links stehenden Indizen $\beta_{i}$ und $\varepsilon_{i}$ verschieden ist. Dann erhält man wegen $a_{\varepsilon} r_{\alpha}=a_{\varepsilon} r=o$

$$
a_{\varepsilon} \cdot a=\sum_{i}^{*} \pi_{i} a_{\alpha_{i}} \notin R,
$$

woraus folgt, daß $R$ quasimodular in $A$ ist.

Es gilt offenbar Rang $A=\operatorname{Rang} R=1$ (über $K_{p}$ ).

Somit ist der Satz bewiesen.

\section{$\S 3$. Untersuchung der $\Omega$-Ringe}

In diesem und den folgenden $\S$ werden wir die $\Omega$-Ringe (welche die Lösungen des im $\S 1$ erwähnten Problems von A. KERTÉsz sind), im allgemeinen untersuchen und auch einige weitere offene Fragen bezüglich der $\Omega$-Ringe erwähnen. ${ }^{5}$

SATz 3. 1. Ein ausgezeichnetes Rechtsideal $R$ eines $\Omega$-Ringes $A$ ist kein Ideal von $A$, und daher ist jeder $\Omega$-Ring nichtkommutativ.

BeweIS. Ist $R$ ein Ideal im $\Omega$-Ring $A$, so hat der Faktorring $A / R$ kein nichttriviales Rechtsideal, und somit ist $A / R$ entweder ein Schiefkörper oder ein Zeroring von Primzahlordnung. Ist $A / R$ ein Schiefkörper, so ist $R$ modular in $A$, und ist $A / R$ ein Zeroring, so ist $R$ gewiß nicht quasimodular in $A$. Da aber $R$ ein ausgezeichnetes Rechtsideal ist, ist $R$ gewiß kein Ideal in $A$. Freilich ist dann $A$ kein kommutativer Ring, w.z.b.w.

SATZ 3. 2. Ein $\Omega$-Ring $A$ ist kein halbpimärer Ring und somit ist $A$ kein Artinscher Ring.

Beweis. Nach dem Satz von Kertész [8] gilt $J \subseteq R$ für das Radikal $J$ und für jedes ausgezeichnete Rechtsideal $R$ von $A$, denn $\bar{J}$ ist der Durchschnitt aller ausgezeichneten Rechtsideale. Dann ist $A / J$ ebenfalls ein $\Omega$-Ring. Ist nun $A / J$ ein Artinscher Ring, so besitzt $A / J$ ein Einselement, woraus der Widerspruch folgt, daß $R$ modular in $A$ ist. Also ist $A / J$ für einen $\Omega$-Ring $A$ kein Artinscher Ring.

Satz 3. 3. Jeder $\Omega$-Ring A besitzt ein homomorphes Bild, der ein halbeinfacher und bezüglich eines ausgezeichneten Rechtsideales $R$ reduzierter $\Omega$-Ring ist.

${ }^{5}$ Die Klasse der nicht $\Omega$-Ringe ist eine gewissermaßen ähnliche Verallgemeinerung der kommutativen Ringe und der Artinschen Ringe, wie auch die Klasse der FC-Gruppen (d. h. Gruppen mit endlichen Klassen von konjugierten Elementen) eine Verallgemeinerung der kommutativen Gruppen und der endlichen Gruppen ist. 
BeweIs. Es sei $K$ die Summe derjenigen Ideale $I$ von $A$, die im Rechtsideal $R$ enthalten sind. Dann ist $K$ ein solches Ideal von $A$, daß $A / K$ ein bezüglich $R$ reduzierter $\Omega$-Ring ist. Da nach KeRTÉsz [8] $J \subseteq R$ besteht und $J$ ein Ideal von $A$ ist, ist $A / K$ halbeinfach.

Lemma 3. 4. Läßt sich ein Rechtsideal der Gestalt $R=(1-x) A=\{a-x a ; a \in A\}$ durch ein idempotentes Element e erzeugen, d.h. gilt $R=e A$, so enthält $A$ ein Linkseinselement.

Beweis. Wegen $(1-x) A=e A$ erhält man $(1-e)(1-x) A=o$, folglich $y=(e+x-e x) y$ für jedes $y \in A$, woraus sich ergibt, daß $f=e+x-e x$ ein Linkseinselement von $A$ ist.

SAtz 3. 5. In einem bezüglich $R$ reduzierten $\Omega$-Ring $A$ ist $(1-x) A$ für jedes $x \in A$ kein minimales Rechtsideal.

BeweIS. Wegen der Reduziertheit ist $A$ halbeinfach (vgl. dem Beweis von Satz 3. 4). Ist nun $(1-x) A$ für ein $x \in A$ ein minimales Rechtsideal, so gibt es ein $e \in A$ mit $e^{2}=e \neq 0$ und $(1-x) A=e A$, woraus sich nach dem Lemma 3.4 die Existenz eines Linkseinselementes, folglich die Modularität von $R$ in $A$ ergibt. Das ist aber ein Widerspruch, denn $R$ ist in $A$ ausgezeichnet. Also ist $(1-x) A$ kein minimales Rechtsideal.

SATz 3. 6. Besitzt ein beliebiger $\Omega$-Ring $A$ ein idempotentes Element $e \neq 0$, so gibt es schon in jedem ausgezeichneten Rechtsideal $R_{\alpha}$ ein idempotentes Element $f_{\alpha} \neq 0$.

Beweis. Es gilt $A=R_{\alpha}+(1-e) A$ für jedes $R_{\alpha}$, denn $R_{\alpha}$ ist maximal und nicht modular in $A$. Es gibt also Elemente $r_{\alpha} \in R_{\alpha}$ und $a_{\alpha} \in A$ mit $e=r_{\alpha}+(1-e) a_{\alpha}$ woraus man $e=e r_{\alpha}, e=e r_{\alpha} e,\left(r_{\alpha} e\right)^{2}=r_{\alpha} e \neq o$ und $f_{\alpha}=r_{\alpha} e \in R_{\alpha}$ erhält, w.z.b.w.

SAtz 3. 7. In jedem $\Omega$-Ring $A$ gelten sowohl $a R_{\alpha}+R_{\alpha}=A$ als auch $(1-a) R_{\alpha}+$ $+R_{\alpha}=A$ für jedes ausgezeichnete Rechtsideal $R_{\alpha}$ und für jedes $a \in A$ mit $a \notin R_{\alpha}$.

Es gilt $a R_{\alpha}+(1-a) R_{\alpha}=A$ für jeden $\Omega$-Ring $A$, für jedes ausgezeichnete Rechtsideal $R_{\alpha}$ und für jedes $a \in A$ mit $a \notin R_{\alpha}$.

BEwEIS. Die Menge $S_{\alpha}$ derjenigen Elemente $x \in A$, für die $x A \subseteq R_{\alpha}$ gilt, bildet ein Rechtsideal von $A$ mit $R_{\alpha} \subseteq S_{\alpha} \subseteq A$. Da aber $R_{\alpha}$ quasimodular in $A$ ist, ist $S_{\alpha} \neq A$, woraus wegen der Maximalität von $R_{\alpha}$ in $A$ gewiß $S_{\alpha}=R_{\alpha}$ folgt.

Also erhält man $a A+R_{\alpha}=A$ für jedes $a \in A$ mit $a \oiint R_{\alpha}$. Folglich gibt es Elemente $b_{\alpha} \in A$ und $r_{\alpha} \in R_{\alpha}$ mit $a=a b_{\alpha}+r_{\alpha}$, woraus sich $a\left(1-b_{\alpha}\right) A=r_{\alpha} A \subseteq R_{\alpha}$ ergibt. Da $R_{\alpha}$ maximal und nicht modular in $A$ ist, gewinnen wir $A=R_{\alpha}+\left(1-b_{\alpha}\right) A$, und daher auch $a A \subseteq a R_{\alpha}+R_{\alpha}$ und $a R_{\alpha}+R_{\alpha} \subseteq a A+R_{\alpha} \subseteq a R_{\alpha}+R_{\alpha}$ d.h. $A=a R_{\alpha}+R_{\alpha}$. Ist nun $(1-a) R_{\alpha} \subseteq R_{\alpha}$, so erhält man $a r_{\alpha} \in R_{\alpha}$ für jedes $r_{\alpha} \in R_{\alpha}$, was nach dem obigen nur im Falle $\bar{a} \in R_{\alpha}$ möglich ist. Also besteht $(1-a) R_{\alpha}+R_{\alpha}=A$ für jedes $a \notin R_{\alpha}(a \in A)$ w.z.b.w.

Das Rechtsideal $S=a R_{\alpha}+(1-a) R_{\alpha}$ enthält sowohl $R_{\alpha}$ als auch $a R_{\alpha}$, und somit gilt nach dem ersten Teil von Satz 3.7 gewiß $S=A$, w.z.b.w.

Definition 3. 8. Es sei $A$ ein $\Omega$-Ring und $R_{\alpha}$ ein (festes) ausgezeichnetes Rechtsideal von $A$. Dann sei $R_{\alpha}^{(x)}=\left\{y ; y \in A, x y \in R_{\alpha}\right\}$. 
Offenbar ist $R_{\alpha}^{(x)}$ ein ,Rechtsidealquotient” in $A$, und zwar selbst ein Rechtsideal von $A$.

SATz 3.9. $R_{\alpha}^{(x)}=A$ gilt dann, und nur dann, wenn $x \in R_{\alpha}$ ist. Für $x \notin R_{\alpha}$ bestehen sowohl $R_{\alpha}^{(x)} \nsubseteq R_{\alpha}$ als auch $R_{\alpha} \Phi R_{\alpha}^{(x)}$, und $D_{\alpha}=\bigcap_{x \in A} R_{\alpha}^{(x)}$ ist ein solches zweiseitige Ideal von $A$, für das $A / D_{\alpha}$ ein halbeinfacher, bezüglich $R_{\alpha}$ reduzierter $\Omega$-Ring ist.

BewEIS. Im Falle $R_{\alpha}^{(x)}=A$ besteht $x A \subseteq R_{\alpha}$, folglich $x \in R_{\alpha}$, denn im Falle $x \notin R_{\alpha}$ gilt nach dem Satz 3.7 schon $x R_{\alpha}+R_{\alpha}=A$. Umgekehrt erhält man offenbar $x A \subseteq R_{\alpha}$ für jedes $x \in R_{\alpha}$, folglich ist dann $R_{\alpha}^{(x)}=A$. Also ergibt sich $R_{\alpha}^{(x)} \neq A$ für $x \notin \bar{R}_{\alpha}$. Wenn wir zeigen, daß für $x \notin R_{\alpha} R_{\alpha}^{(x)} \nsubseteq R_{\alpha}$ ist, so folgt daraus auch $R_{\alpha}^{(x)} \nsupseteq R_{\alpha}$. Wie wir bei dem Beweis des Satzes 3.7 schon gesehen haben, gibt es zu jedem $R_{\alpha}$ und jedem $a \in A$ mit $a \notin R_{\alpha}$ ein Element $b_{\alpha}$ mit $a-a b_{\alpha} \in R_{\alpha}$. Da aber $R_{\alpha}$ nicht modular in $A$ ist, existiert ein $y_{\alpha} \in A$ mit $y_{\alpha}-a y_{\alpha} \notin R_{\alpha}$. Wegen $a\left(y_{\alpha}-b_{\alpha} y_{\alpha}\right) \in R_{\alpha}$ gilt $y_{\alpha}-b_{\alpha} y_{\alpha} \in R_{\alpha}^{(a)}$, also auch $R_{\alpha}^{(a)} \nsubseteq R_{\alpha}$. Es sei nun $d$ ein beliebiges Element von $D_{\alpha}=\bigcap_{x \in A} R_{\alpha}^{(x)}$. Dann gilt $x d \in R_{\alpha}$ für jedes $x \in A$, also $A d \subseteq R_{\alpha}$. Da $R_{\alpha}$ quasimodular in $A$ ist, erhält man $d \in R_{\alpha}$, folglich $D_{\alpha} \subseteq R_{\alpha}$. Wegen der Definition von $D_{\alpha}$ folgt aus $A y \subseteq R_{\alpha}$ trivial $y \in D_{\alpha}$, und wegen $\bar{A}^{2} D_{\alpha} \subseteq A D_{\alpha} \subseteq R_{\alpha}$ auch $A D_{\alpha} \subseteq D_{\alpha}$. Somit ist $D_{\alpha}$ ein Ideal von $A$, das in $R_{\alpha}$ liegt. Ist nun $\bar{I}$ ein Ideal von $A$ mit $I \nsubseteq D_{\alpha}$, so gibt es ein Element $f \in I$ mit $f \notin D_{\alpha}$, und somit ein $x \in A$ mit $f \notin R_{\alpha}^{(x)}$. Dann besteht $x f \notin R_{\alpha}$, was wegen $x f \in I$ bedeutet, daß $I \nsubseteq R_{\alpha}$ ist. Also gilt entweder $I \subseteq D_{\alpha}$ oder $I \Phi R_{\alpha}$ für jedes zweiseitige Ideal $I$ von $A$. Hiernach ist $A / D_{\alpha}$ wirklich ein bezüglich $R_{\alpha}$ reduzierter und somit halbeinfacher $\Omega$-Ring, w.z.b.w.

SATz 3. 10. Sind $A$ ein $\Omega$-Ring mit einem ausgezeichneten Rechtsideal $R_{\alpha}, D_{\alpha}=$ $=\bigcap_{x \in A} R_{\alpha}^{(x)}$ und $R_{1}$ ein Rechtsideal von $A$ mit $R_{1} \nsubseteq D_{\alpha}$, so gibt es ein a $A \in A$ mit $a \ddagger R_{\alpha}$ derart, daß $a R_{1}+R_{\alpha}=A$ gilt.

BEweIS. Wegen $R_{1} \Phi D_{\alpha}$ existiert ein $r_{1} \in R_{1}$ mit $r_{1} \notin D_{\alpha}$, folglich ein $a \in A$ mit $r_{1} \notin R_{\alpha}^{(a)}$, woraus sich $a r_{1} \notin R_{\alpha}$ ergibt. Da $a R_{1} \nsubseteq R_{\alpha}$ besteht, erhält man wegen der Maximalität von $R_{\alpha}$ in $A$ die Gleichung $a R_{1}+R_{\alpha}=A$, w.z.b.w.

SATz 3. 11. Ist A ein bezüglich eines ausgezeichneten Rechtsideales $R_{\alpha}$ reduzierter $\Omega$-Ring, so ist $(o)_{l}$ das einzige in $R_{\alpha}$ liegende Linksideal von $A$.

Beweis. Ist $L$ ein Linksideal von $A$ mit $L \subseteq R_{\alpha}$, so gilt wegen $L A \subseteq R_{\alpha}$ und der Reduziertheit von $A$ bezüglich $R_{\alpha} L A=o$, denn $L A$ ist ein Ideal in $R_{\alpha}$. Da aber $A$ wegen der Reduziertheit halbeinfach ist, ergibt sich $L=o$, w.z.b.w.

SATz 3. 12. Sowohl der Rechtsannullator $A_{r}$ als auch der Linksannulator $A_{l}$ eines ausgezeichneten Rechtsideales $R_{\alpha}$ eines bezüglich $R_{\alpha}$ reduzierten $\Omega$-Ringes A stimmen mit (o) überein.

Beweis. Ist $x \in A_{r}$, so gilt $R_{\alpha} x=0$, folglich $A x=0$, denn man nach Satz 3. 7 $y R_{\alpha}+R_{\alpha}=A$ für jedes $y \notin R_{\alpha}(y \in A)$ erhält. Wegen der Halbeinfachheit von $A$ folgt aus $A x=0$ trivial $x=o$. Ist nun $z \in A_{l}$ so gilt $z R_{\alpha}=o$, folglich $z \in R_{\alpha}$ nach dem Satz 3. 7, denn im Falle $z \notin R_{\alpha}$ würde $z R_{\alpha} \subseteq R_{\alpha}$ und somit $z R_{\alpha} \neq 0$ bestehen. Also gilt $A_{l} \subseteq R_{\alpha}$, und da $A$ ein Linksideal von $A$ in $R_{\alpha}$ ist, folgt wegen der Reduziertheit von $\bar{A}$ bezüglich $R_{\alpha} A_{l}=o$, w.z.b.w. 
SATz 3.13. Es sei $A$ ein bezüglich eines ausgezeichneten Rechtsideales $R_{\alpha}$ reduzierter $\Omega$-Ring. Dann gilt $R_{\alpha} e \neq R_{\alpha}$ für jedes idempotente Element e von A. Weiterhin folgt $x=0$ sowohl aus $R_{\alpha}^{k} x=o$ als auch aus $x R_{\alpha}^{k}=o$ für jeden Exponenten $k$.

BEWEIS. Ist $R_{\alpha} e=R_{\alpha}$ für ein idempotentes Element $e \in A$, so ergibt sich wegen $a R_{\alpha}+R_{\alpha}=A$ für jedes $a \notin R_{\alpha}(a \in A)$ gewiß $A e=A$. Also ist $e$ ein Rechtseinselement von $A$, und da $A$ halbeinfach ist, ist $e$ ein zweiseitiges Einselement in $A$. Da aber $R_{\alpha}$ nicht modular in $A$ ist, hat $A$ kein Linkseinselement. Es gilt also $R_{\alpha} e \neq R_{\alpha}$. Ist nun $x R_{\alpha}^{k}=o\left(R_{\alpha}^{k} x=o\right)$, so ergibt sich $x R_{\alpha}^{k-1} \subseteq A_{l}\left(R_{\alpha}^{k-1} x \subseteq A_{r}\right)$ und wegen $A_{l}=o$ $\left(A_{r}=o\right)$ (vgl. Satz 3.13) $x R_{\alpha}^{k-1}=o\left(R_{\alpha}^{k-1} x=o\right)$. Nach endlich vielen Schritten erhält man $x R_{\alpha}=o\left(R_{\alpha} x=o\right)$, folglich $x=o$ nach dem Satz 3. 13, w.z.b.w.

SAtz 3. 14. Es seien A ein bezüglich eines ausgezeichneten Rechtsideales $R_{\alpha}$ reduzierter $\Omega$-Ring und $R_{1}$ ein Rechtsideal von A mit $R_{1} \Phi R_{\alpha}$, weiterhin $D_{\alpha}^{\prime}=\bigcap_{x \in R_{1}} R_{\alpha}^{(x)}=$ $=\left\{y ; R_{1} y \subseteq R_{\alpha}\right\}$. Dann gilt $D_{\alpha}^{\prime}=o$. Ist nun $I_{\alpha}^{(x)}=\left\{y ; R_{\alpha} y \subseteq R_{\alpha}^{(x)}\right\}$, so ergibt sich $I_{\alpha}^{(x)}=o$. Ferner ist $(o)$ das einzige in $R_{\alpha}^{(x)}$ liegende zweiseitige Ideal von $A$.

Beweis. Wegen $R_{1}+R_{\alpha}=A$ und wegen $R_{1} D_{\alpha}^{\prime} \sqsubseteq R_{\alpha}$ erhält man $A D_{\alpha}^{\prime} \subseteq R_{\alpha}$, folglich $D_{\alpha}^{\prime}=o$, weil $A, D_{\alpha}^{\prime}$ ein zweiseitiges in $R_{\alpha}$ liegendes Ideal von $A, A$ reduziert und halbeinfach ist. Wegen $R_{\alpha} I_{\alpha}^{(x)} \subseteq R_{\alpha}^{(x)}$ und $R_{\alpha}^{(x)}+R_{\alpha}=A$ (vgl. Satz 3.10) gilt $A I_{\alpha}^{(x)} \subseteq R_{\alpha}^{(x)}$, folglich $x A I_{\alpha}^{(x)} \subseteq R_{\alpha}$, und wegen $x R_{\alpha}+R_{\alpha}=A$ (für jedes $x \in A, x \notin R_{\alpha}$ ) ergibt sich $A I_{\alpha}^{(x)} \subseteq R_{\alpha}$. Da aber $A I_{\alpha}^{(x)}$ ein Ideal des halbeinfachen reduzierten $\Omega$-Ringes $A$ ist, erhält man $A, I_{\alpha}^{(x)}=o$ und somit $I_{\alpha}^{(x)}=o$. Es sei $I$ ein Ideal von $A$ in $R_{\alpha}^{(x)}$. Wegen $A I \subseteq R_{\alpha}^{(x)}$ gilt dann $x A I \subseteq R_{\alpha}$, folglich wegen $x R_{\alpha}+R_{\alpha}=A$ (vgl. Satz 3. 7) auch $A I \subseteq R_{\alpha}$, woraus sich $A I=O$ und $I=0$ ergibt.

SAtz 3. 15. Es seien $A$ ein bezüglich eines ausgezeichneten Rechtsideales $R_{\alpha}$ reduzierter $\Omega$-Ring, $x$ ein Element von $A$ mit $x \notin R_{\alpha}$ und $R_{1}$ ein Rechtsideal von $A$ mit $R_{1} \Phi R_{\alpha}^{(x)}$. Dann gelten $\left(x \dot{R}_{1}\right)^{k}+R_{\alpha}=x R_{1}^{k}+R_{\alpha}=(1-x) R_{\alpha}^{k}+R_{\alpha}=A$ für jeden Exponenten $k$. Im Falle $o \neq R_{1}^{k} \subseteq R_{\alpha}$ besteht auch $(1-x) R_{1}^{k}+R_{\alpha}=A$. Ist $K_{\alpha}^{(x, k)}=$ $=\left\{y ; y \in A, R_{1}^{k} y \leqq R_{\alpha}^{(x)}\right\}$, so gilt $\bar{K}_{\alpha}^{(x, k)}=o$ für jeden Exponenten $k$.

BEWEIS. Nehmen wir an, daß $\left(x R_{1}\right)^{k+1} \subseteq R_{\alpha}$ und $\left(x R_{1}\right)^{k} \nsubseteq R_{\alpha}$ ist. Dann gilt wegen $R_{1} \subseteq R_{\alpha}^{(x)}$ offenbar $x R_{1} \subseteq R_{\alpha}$ und somit wegen der Maximalität von $R_{\alpha}$ in $A$ auch $x R_{1}+R_{\alpha}=A$. Daher gilt $k \geqq 1$. Nach der Voraussetzung besteht $\left(x R_{1}\right)^{k}+R_{\alpha}=$ $=A$, und wegen $\left(x R_{1}\right)^{k+1} \subseteq R_{\alpha}$ auch $A x R_{1} \subseteq R_{\alpha}$. Da $A$ reduziert und halbeinfach ist, ergibt sich $A x R_{1}=o$ und $x R_{1}=o$, obwohl nach der Voraussetzung $\left(x R_{1}\right)^{k} \nsubseteq R_{\alpha}$ und $k \geqq 1$ bestehen. Also gilt $\left(x R_{1}\right)^{k}+R_{\alpha}=A$ für jedes $k \geqq 1$. Wegen $\left(x R_{1}\right)^{k} \subseteq x R_{1}^{k}$ erhält man auch $x R_{1}^{k}+R_{\alpha}=A$ für jedes $k \geqq 1$. - Wegen $R_{1} \Phi R_{\alpha}^{(x)}$ besteht $x R_{1}^{k} \bar{\Phi} R_{\alpha}$. Folglich gibt es ein $r_{1} \in R_{1}^{k}$ mit $x r_{1} \notin R_{\alpha}$. Ist nun $(1-x) R_{1}^{k} \subseteq R_{\alpha}$, so erhält man im Falle der Voraussetzung $R_{1}^{k} \subseteq R_{\alpha}$ auch $x R_{1}^{k} \subseteq R_{\alpha}$, was der Bedingung $x r_{1} \notin R_{\alpha}$ widerspricht. Also folgt aus $R_{1}^{k} \subseteq R_{\alpha}$ und $R_{1} \Phi \overline{R_{\alpha}^{(x)}}$ die Gleichung $(1-x) R_{1}^{k}+R_{\alpha}=A$.

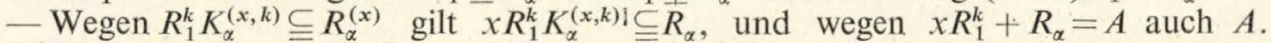
$K_{\alpha}^{(x, k)} \subseteq R_{\alpha}$, woraus $K_{\alpha}^{(x, k)}=0$ folgt, denn $A, K_{\alpha}^{(x, k)}$ ist ein Ideal von $A$ in $R_{\alpha}$, und $A$ ist reduziert und halbeinfach.

Bemerkung 3.16. Die Voraussetzungen des Satzes 3.16 bezüglich $R_{1}$ gelten freilich auch für $R_{\alpha}$ selbst. Also gelten auch $\left(x R_{\alpha}\right)^{k}+R_{\alpha}=x R_{\alpha}^{k}+R_{\alpha}=(1-x) R_{\alpha}^{k}+$ $+R_{\alpha}=A$ für jedes $k \geqq 1$. Mit Hilfe einer vollständigen Induktion nach $k$ kann auch $(A x)^{k} \nsubseteq R_{\alpha}$ für jeden bezüglich eines ausgezeichneten Rechtsideales $R_{\alpha}$ reduzierten $\Omega$-Ring $A$ und für jedes $k \geqq 1$ bewiesen werden. 


\section{$\S 4$. Fortsetzung der Untersuchung der $\Omega$-Ringe}

Wir betrachten hier weitere Eigenschaften der $\Omega$-Ringe. Es gilt der folgende

SAtz 4. 1. Das Zentrum $Z$ jedes bezüglich eines ausgezeichneten Rechtsideales $R_{\alpha}$ reduzierten $\Omega$-Ringes $A$ ist $\{o\}$.

BEweIS. Ist $z$ ein Element des Zentrums $Z$ von $A$, so gilt wegen $z R_{\alpha}=R_{\alpha} z \subseteq R_{\alpha}$ und nach dem Satz 3.7 offenbar $z \in R_{\alpha}$, denn im Falle $z \notin R_{\alpha}$ würde $z R_{\alpha} \Phi R_{\alpha}$ bestehen. Wegen $Z \subseteq R_{\alpha}$ ist dann $I=Z A=A Z$ ein in $R_{\alpha}$ liegendes Ideal von $A$, woraus $Z A=o$ und $Z=\{o\}$ folgt, weil $A$ reduziert und halbeinfach ist.

Satz 4. 2. Die additive Gruppe $A^{+}$eines bezüglich eines ausgezeichneten Rechtsideales $R_{\alpha}$ reduzierten $\Omega$-Ringes $A$ ist entweder eine elementare $p$-Gruppe (d.h. es gilt $p A=o)$, oder $A^{+}$ist torsionsfrei mit $A^{+}=n A^{+}+R_{\alpha}$ für jede von Null verschiedene ganze rationale Zahl $n$, weiterhin ist $R_{\alpha}^{+}$eine reine (servante) Untergruppe der Gruppe $A^{+}$.

BeweIs. Es sei $A[p]$ für jede Primzahl $p$ die Menge aller Elemente $x$ der Ordnung $p$, d.h. mit $p x=o$. Ist $A^{+}$nicht torsionsfrei, so existiert ein $p$ mit $A[p] \neq o$. Da $A[p]$ ein Ideal von $A, A$ reduziert und $R_{\alpha}$ maximal in $A$ ist, gilt $A=A[p]+R_{\alpha}$. Hiernach ist $p A=p R_{\alpha} \subseteq R_{\alpha}$. Da aber $A$ bezüglich $R_{\alpha}$ reduziert und $p A$ ein Ideal von $A$ in $R_{\alpha}$ ist, gilt $p A=o$. Ist aber $A^{+}$torsionsfrei, so gilt $n A \neq 0$ für jede von Null verschiedene ganze rationale Zahi $n$, woraus wegen $n A \nsubseteq R_{\alpha}$ trivial $A=n A+R_{\alpha}$ folgt. - Im Falle $p A=o$ ist $R_{\alpha}^{+}$ein direkter Summand, also auch eine reine Untergruppe von $A^{+}$. Im torsionsfreien Falle besteht $a \notin R_{\alpha}$ aber $n a \in R_{\alpha}$ für eine von Null verschiedene ganze rationale Zahl $n$. Dann gilt aber $a R_{\alpha}+R_{\alpha}=A$, woraus $n A \subseteq R_{\alpha}$ folgt, was der Reduziertheit von $A$ bezüglich $R_{\alpha}$ widerspricht, denn $n A$ ist ein Ideal von $A$ in $R_{\alpha}$. Also ist $R_{\alpha}^{+}$im beiden Fällen eine reine Untergruppe in $A^{+}$.

BEMERKUNG 4. 3. Ist $A$ ein $\Omega$-Ring, der bezüglich eines ausgezeichneten Rechtsidea's $R_{\alpha}$ reduziert ist, so ist $A^{+}$nach dem Satz 4. 2 dann und nur dann teilbar (divisible), wenn $R_{\alpha}^{+}$teilbar ist.

SATz 4. 4. Es seien $R_{\alpha}$ ein ausgezeichnetes Rechtsideal eines $\Omega$-Ringes $A$, der bezüglich $R_{\alpha}$ nicht notwendig reduziert ist, und $x$ ein solches Element von $A$, für welches $x \notin R_{\alpha}$ und $x R_{\alpha}^{(x)}=R_{\alpha}$ bestehen. Dann ist $x$ ein Linksteiler jedes Elements $a \in A$, aber kein Linkseinselement von $A$, und es gilt $x R=x A=A$. Weiterhin ist der Unterring $\{x\}$ unendlich, es gilt $f(x) R_{\alpha}+R_{\alpha}=A$ für jedes Element $o \neq f(x) \in\{x\}$, und es gibt ein Element $y$ von $A$ mit $y \notin R_{\alpha}$ und mit $(y-f(x)) R_{\alpha}+R_{\alpha}=A$ für jedes $f(x) \in\{x\}$.

BEweIs. Wegen $R_{\alpha}^{(x)} \nsubseteq R_{\alpha}$ und der Maximalität von $R_{\alpha}$ in $A$ ist $R_{\alpha}^{(x)}+R_{\alpha}=A$, woraus man wegen $x R_{\alpha}^{(x)} \subseteq R_{\alpha}$ offenbar $R_{\alpha}+x R_{\alpha}=x A$ erhält. Da aber $R_{\alpha}+x R_{\alpha}=A$ für jedes $x \notin R_{\alpha}$ gilt, ergibt sich $x A=A$. Folglich gibt es eine Lösung $a$ jeder Gleichung $x a=b(a, b \in A)$, wenn $x R_{\alpha}^{(x)}=R_{\alpha}$ ist. Es sei $c$ ein Element von $A$ mit $x c=x$. Dann ergibt sich $A=R_{\alpha}+(1-c) A$, denn $R_{\alpha}$ ist maximal und nicht modular in $A$. Wegen $x(1-c)=o$ gilt hiernach $x A=x R_{\alpha}=A . x$ ist also ein Linksteiler jedes Elementes $a$ von $A$, aber kein Linkseinselement von $A$, denn $R_{\alpha}$ ist nicht modular in $A$. Es gilt dann $x^{n} R_{\alpha}=A$ für jede natürliche Zahl $n$. Gibt es nun Exponenten $m$ und $n$ mit $m \neq n$ und $x^{m}=x^{n}$, so existiert ein idempotentes Element $e=x^{k}$ in der Menge aller 
Potenzen von $x$ (vgl. z.B. RÉDEI's Buch [9]), und dann ist $e$ ein Linkseinselement von $A=x^{k} A=e A$, was unmöglich ist, denn $R_{\alpha}$ ist in $A$ nicht modular. Im Falle $m \neq n$ gilt also $x^{m} \neq x^{n}$, und somit ist der Unterring $\{x\}$ unendlich. Nehmen wir jetzt an, daß $f(x) \in R_{\alpha}$ für ein $f(x) \in\{x\}$ mit $f(x) \neq 0$ gilt. So besteht

$$
k_{0} x^{i}+k_{1} x^{i+1}+\ldots+k_{n} x^{i+n} \in R_{\alpha}
$$

wobei $k_{j} \in I$, und $I$ der Ring der ganzen, rationalen Zahlen ist. Im Falle $p A=o$ für eine Primzahl $p$ läßt sich offenbar $k_{0} \equiv 1(\bmod p)$ annehmen, woraus

$$
(1+g(x)) x^{i} \in R_{\alpha} \quad(g(x) \in\{x\})
$$

folgt. Dann ist aber $R_{\alpha}$ wegen $x^{i} A=A$ und $(1+g(x)) A \subseteq R_{\alpha}$ modular in $A$, was unmöglich ist. Im Falle $p A=0$ gilt daher $f(x) R_{\alpha}+R_{\alpha}=A$ für jedes $f(x)(\in\{x\}, \neq 0)$. Ist aber $A^{+}$torsionsfrei, so ergibt sich nach dem Satz 4. $2 A=n A+R_{\alpha}$ für jede ganze Zahl $n \neq 0$. Da sich $k_{0}$ in (*) offenbar als von Null verschieden annehmen läßt, gilt auch $A=k_{0} A+R_{\alpha}$ und somit $x^{j}=k_{0} y_{j}+r_{j}$ mit $y_{j} \in A$ und $r_{j} \in R_{\alpha}$ $(j=1,2, \ldots, n)$, folglich

$$
k_{0}(1+y) x^{i} \in R_{\alpha} \quad(y \in A) .
$$

Da aber $R_{\alpha}^{+}$nach dem Satz 4. 2 eine reine Untergruppe in $A^{+}$ist, ergibt sich wegen $k_{0} \neq o$ auch

$$
(1+y) x^{i} \in R_{\alpha},
$$

was wegen $x^{i} A=A$ und $(1+y) A \subseteq R_{\alpha}$ genau die Modularität des Rechtsideales $R_{\alpha}$ in $A$ bedeutet. Letzteres ist aber unmöglich, und dieser Widerspruch beweist $f(x) R_{\alpha}+R_{\alpha}=A$ auch im torsionsfreien Falle $\left(f(x) \in\{x\}, f(x) \neq 0, x R_{\alpha}^{(x)}=R_{\alpha}\right)$. Nehmen wir an, daß es zu jedem $y \in A$ ein Element $f(x) \in\{x\}$ mit $y-f(x) \in R_{\alpha}$ gibt. Aus dieser Voraussetzung werden wir einen Widerspruch ableiten. Im Falle $y-f(x) \in R_{\alpha}$ ergibt sich nämlich $y=f(x)+r$ mit $r \in R_{\alpha}$. Da wegen $x R_{\alpha}=A$ ein Element $r^{*} \in R_{\alpha}$ mit $x r^{*}=x$ existiert, erhält man nach der Voraussetzung für ein beliebiges $y \in A$ die Beziehung $y\left(1-r^{*}\right) x=(f(x)+r)\left(1-r^{*}\right) x=r\left(1-r^{*}\right) x$, folglich $A\left(1-r^{*}\right) A=A\left(1-r^{*}\right) x A \subseteq R_{\alpha}$. Aus $A\left(1-r^{*}\right) A \subseteq R_{\alpha}$ ergibt sich wegen der Quasimodularität von $R_{\alpha}$ in $A$ gewiß $\left(1-r^{*}\right) A \subseteq R_{\alpha}$, denn es gilt $A z \subseteq R_{\alpha}$ für jedes $z \neq R_{\alpha}$ $(z \in A)$. Da aber $R_{\alpha}$ nicht modular in $A$ ist, gilt $\left(1-r^{*}\right) A \Phi R_{\alpha}$, und somit ist $\left(1-r^{*}\right) A \subseteq$ $\subseteq R_{\alpha}$ ein Widerspruch. Also gibt es ein $y \in A$ mit $(y-f(x)) R_{\alpha}+R_{\alpha}=A$ für jedes $\overline{f(x)} \in\{x\}$ w.z.b.w.

Am Ende dieses Paragraphen möchten wir einige Probleme aufwerfen.

Problem 1. Gibt es einen $\Omega$-Ring $A$, der sich als Ring, durch ein ausgezeichnetes Rechtsideal $R$ und durch endlich viele Elemente $a_{1}, a_{2}, \ldots, a_{n}$ erzeugen läßt?

Problem 2. Gibt es einem $\Omega$-Ring $A$ mit einem ausgezeichneten Rechtsideal $R$ und einer endlichen Teilmenge $a_{1}, a_{2}, \ldots, a_{n}$ von $A$, derart, daß für jedes $x \in A$ mit $x \notin R$ eine der Relationen $a_{i} x \notin R(i=1,2, \ldots, n)$ gilt? (Dasselbe Problem auch für $n=2$.)

Problem 3. Gibt es einen $\Omega$-Ring, der ein MHR-Ring ist, d.h. in dem die Minimalbedingung für Hauptrechtsideale gilt? (vgl. Verfasser [11]). 
Problem 4. Man untersuche in einem $\Omega$-Ring den Zusammenhang zwischen der Mächtigkeit $m$ der Menge aller modularen maximalen Rechtsideale und der Mächtigkeit $n$ der Menge aller ausgezeichneten Rechtsideale!

\section{$\S 5$. Weitere Bemerkungen über das Jacobsonsche Radikal}

Die Resultate dieses Paragraphen sind Folgerungen des Satzes von KERTÉsz [7].

Ein Teil der hier erwähnten Sätze ist nicht ganz neu, aber wir betrachten in diesem $\S$ auch solche, schon bekannte Ergebnisse, die sich mit der Hilfe des Frattinischen Untermoduls $\Phi_{r}$ eines Ringes (vgl. Fußnote 3) eleganter beweisen lassen.

Es gilt der folgende

SATz 5. 1. Es sei A ein assoziativer Ring. Gilt $\Phi_{r} \subseteq R \subseteq J$ für ein Rechtsideal $R$, für den Frattinischen Untermodul $\Phi_{r}$ des als A-Rechtsmodul aufgefassten Ringes $A$ und für das Jacobsonsche Radikal $J$, so ist $R$ ein zweiseitiges Ideal von $A$.

Beweis. Nach Hille [4] bzw. Kertész [7] gilt $A J \subseteq \Phi_{r}$, folglich auch $A R \subseteq$ $\subseteq \Phi_{r} \subseteq R$, w.z.b.w.

SATz 5. 2. Sind für die natürlichen Zahlen $m, n(\geqq 1)$ die Mengen $I_{m}, K_{n}, L_{m, n}$ in einem Ring A folgendermaßen definiert:

$$
\begin{gathered}
I_{m}=\left\{x ; x \in A, A^{m} x \subseteq \Phi_{r}\right\}, \\
K_{n}=\left\{y ; y \in A, y A^{n} \subseteq \Phi_{l}\right\}, \\
L_{m, n}=\left\{z ; z \in A, A^{m} z A^{n} \subseteq M\right\}
\end{gathered}
$$

wobei $M=\Phi_{r}$ oder $\Phi_{l}$ bzw. $\Phi_{r} \cap \Phi_{l}$, so gilt

$$
J=I_{m}=K_{n}=L_{m, n}
$$

für das Jacobsonsche Radikal J von A.

BEwEIS. Wegen der Ähnlichkeit der Beweisteile, werden wir nur $J=L_{m, n}$ beweisen. Nach Hille [4] bzw. KeRTész [7] gilt offenbar $J \subseteq L_{m, n}$. Ist nun $z \in L_{m, n}$, und ist z.B. $M=\Phi_{r} \cap \Phi_{l}$, so bestehen sowohl $A^{m} z A^{n} \subseteq \Phi_{r}$ als auch $A^{m} z A^{n} \subseteq \Phi_{l}$, woraus man nach [7] $A^{m-1} z A^{n} \subseteq J$ bzw. $A^{m} z A^{n-1} \subseteq J$ erhält. Da aber $A / J$ halbeinfach ist, hat $A / J$ keinen von $\bar{N}$ ull verschiedenen linksseitigen oder rechtsseitigen Annullator, deshalb ergibt sich $A^{m-1} z A^{n-1} \subseteq J$ und nach endlich vielen ähnlichen Schritten $A z \subseteq J$ bzw. $z A \subseteq J$, woraus $z \in J$ und somit $L_{m, n} \subseteq J$ folgt. Es ist also $J=L_{m, n}$.

SATZ 5. 3. Ein Ring $A$ ist dann und nur dann ein Radikalring im Sinne von Jacobson, wenn der Faktorring $A / \Phi_{r}$ von A nach dem Frattinischen Untermodul $\Phi_{r}$ des als A-Rechtsmodul betrachteten Ringes A, ein Zeroring ${ }^{6}$ ist. Gilt in einem Ring $A$ die Bedingung $\Phi_{r}=o$, so enthält das Jacobsonsche Radikal $J$ von A nur die Rechts-

${ }^{6}$ Ein Ring $A$, in dem das Produkt $x y$ für jedes $x, y \in A$ Null ist, heißt Zeroring. 
annullatoren von A. Ist A ein von Null verschiedener einfacher Radikalring ${ }^{7}$ mit $A^{2}=A$, so besitzt A kein maximales echtes Rechtsideal, also gilt $\Phi_{r}=A$.

BEWEIS. Ist $J=A$, so erhält man wegen $A J \subseteq \Phi_{r}$ die Inklusion $A^{2} \subseteq \Phi_{r}$, und somit ist dann $A / \Phi_{r}$ ein Zeroring. Ist umgekehrt $A / \Phi_{r}$ ein Zeroring, so gilt $\bar{A}^{2} \subseteq \Phi_{r}$, woraus sich nach KeRTÉsz [7] gewiß $A=J$ ergibt. - Besteht nun $\Phi_{r}=o$, so enthält $J$ wegen $A J \subseteq \Phi_{r}$ nur die Rechtsannullatoren von $A$. - Hat $A$ ein maximales echtes Rechtsideal, so gilt $\Phi_{r} \neq A$. Ist nun $A$ insbesondere ein einfacher Radikalring, so ergibt sich $\Phi_{r}=o$, denn $\Phi_{r}$ ist ein Ideal mit $\Phi_{r} \neq A$. Da $A^{2} \subseteq \Phi_{r}$ nach dem obigen für jeden Radikalring gilt, erhält man $A^{2}=o$, was wegen $\overline{A^{2}}=A$ den Widerspruch $A=o$ bedeutet. Also hat jeder von Null verschiedene einfache Radikalring $A$ mit $A^{2}=A$ kein maximales echtes Rechtsideal, w.z.b.w.

Satz 5. 4. Gilt $J^{2} \neq 0$ für das Jacobsonsche Radikal J eines beliebigen Ringes A, so besitzen der rechtsseitige Frattinische Untermodul $\Phi_{r}$ und der linksseitige Frattinische Untermodul $\Phi_{l}$ des A-Rechtsmoduls bzw. A-Linksmoduls A einen von Null verschiedenen Durchschnitt.

BEwEIs. Nehmen wir an, daß $\Phi_{r} \cap \Phi_{l}=o$ ist. Dann erhält man wegen $A J \subseteq \Phi_{r}$ und $J A \subseteq \Phi_{l}$ offenbar $J^{2} \subseteq \Phi_{r}$ und $J^{2} \subseteq \Phi_{l}$, folglich $J^{2} \subseteq \Phi_{r} \cap \Phi_{l}=o$. Es ergibt sich also im Falle $J^{2} \neq 0$ notwendigerweise $\Phi_{r} \cap \Phi_{l} \neq 0$.

BemerkUNGen 5. 5. In jedem kommutativen Ring $A$ mit $\Phi_{r} \neq 0$ ist trivial $\Phi_{r} \cap \Phi_{l}=\Phi_{r}=\Phi_{l} \neq o$. Jeder Matrizenring über einem Schiefkörper, der offenbar halbeinfach ist, ist ein Ring, für den $J^{2}=J=o$ und $\Phi_{r}=\Phi_{l}=\Phi_{r} \cap \Phi_{l}=o$ bestehen.

Es gibt aber auch nichthalbeinfache Ringe, und zwar schon solche mit sechszehn Elementen, für die $o \neq \Phi_{r} \neq \Phi_{l} \neq o, \Phi_{r} \cap \Phi_{l}=o$ und $J=\Phi_{r}+\Phi_{l}$ mit $J^{2}=o$ gelten. Ein solcher Ring ist z.B. die Algebra $A=\left\{a_{1}, a_{2}, b_{1}, b_{2}\right\}$ mit $2 A=o$ (d.h. $x+x=o$ für jedes $x \in A$ ) und mit der Multiplikationstabelle

\begin{tabular}{|c|c|c|c|c|}
\cline { 2 - 5 } & $a_{1}$ & $a_{2}$ & $b_{1}$ & $b_{2}$ \\
\hline$a_{1}$ & $a_{1}$ & $o$ & $o$ & $o$ \\
\hline$a_{2}$ & $o$ & $a_{2}$ & $o$ & $b_{2}$ \\
\hline$b_{1}$ & $b_{1}$ & $o$ & $o$ & $o$ \\
\hline$b_{2}$ & $o$ & $o$ & $o$ & $o$ \\
\hline
\end{tabular}

In diesem Ring $A$ gelten wegen $\Phi_{r}=\left\{b_{2}\right\}$ und $\Phi_{l}=\left\{b_{1}\right\}$ die Beziehungen $\Phi_{r} \cap \Phi_{l}=o$ und $J^{2}=o$ für das Radikal $J=\Phi_{r}+\Phi_{l}$.

Es sei $B$ die durch $a_{1}$ und $b_{1}$ erzeugte Teilalgebra der obigen Algebra $A=\left\{a_{1}, a_{2}, b_{1}, b_{2}\right\}$. Im Ring $B=\left\{a_{1}, b_{1}\right\}$ gilt $B^{2}(=B) \Phi R$ für jedes maximale Rechtsideal $R$ des Ringes $B$, denn $a_{1}$ ist ein Linkseinselement von $B$. Da aber $b_{1} \notin\left\{a_{1}\right\}$ und $x b_{1}=o \in\left\{a_{1}\right\}$ für jedes $x \in B$ bestehen, ist das maximale Rechtsideal $\left\{a_{1}\right\}$ von $B$ nicht quasimodular in $B$.

7 Die Existenz der einfachen Radikalringe $A$ mit $A^{2}=A \neq O$ wurde in SASIADA [10] gezeigt. 
Die Eigenschaft $B^{2}+R=B$ ist also für ein maximales Rechtsideal $R$ eines Ringes $B$ von der Quasimodularität von $R$ in $B$ verschieden.

Ist $A$ zum Schluß ein einfacher Radikalring, so bestehen wegen $J=\Phi_{r}=\Phi_{l}=A$ die Ungleichungen $J^{2} \neq 0$ und $\Phi_{r} \cap \Phi_{l} \neq 0$ (vgl. SASIADA [10]).

(Eingegangen am 22. April 1966.)

\section{Literaturverzeichnis}

[1] L. Fuchs, A remark on the Jacobson radical, Acta Sci. Math. (Szeged), 14 (1952), S. 167-168.

[2] L. FuchS, Abelian Groups (Budapest, 1958, Akadémiai Kiadó).

[3] M. HaLl, The Theory of Groups (New York, 1959, Macmillan).

[4] E. Hille, Functional Analysis and Semi Groups (Providence, 1948, Amer. Math. Soc. Colloq. Publ. Vol. 31).

[5] N. JACOBSON, The radical and semi-simplicity for arbitrary rings, Amer. Jour. Math., 67 (1945), S. $300-320$.

[6] N. JacoBson, Structure of Rings (Providence, 1956, Amer, Math. Soc. Colloq. Publ. Vol. 37).

[7] A. Kertész, A characterization of the Jacobson radical, Proc. Amer. Math. Soc., 14:4 (1963), S. 595-597.

[8] A. KerTész, Über Artinsche Ringe (Budapest, 1966, Akadémiai Kiadó) (im Erscheinen).

[9] L. RéDEI, Algebra, I (Leipzig, 1959, Akademische Verlagsgesellschaft).

[10] E. SasiadA, Solution of the problem of existence of simple radical rings, Bull. Acad. Polon. Sci. Classe III, 9 (1961), S. 257.

[11] F. Szász, Über Ringe mit Minimalbedingung für Hauptrechtsideale. I, Publ. Math. Debrecen, 7 (1960), S. 54 64; II, Acta Math. Acad. Sci. Hung., 12 (1961), S. 417 -439; III, Acta Math. Acad. Sci. Hung., 14 (1963), S. 447-461. 